ELECTRONIC RESEARCH ANNOUNCEMENTS OF THE AMERICAN MATHEMATICAL SOCIETY

Volume 3, Pages 110-113 (October 28, 1997)

S $1079-6762(97) 00033-4$

\title{
A DETERMINISTIC DISPLACEMENT THEOREM FOR POISSON PROCESSES
}

\author{
OLIVER KNILL
}

(Communicated by Mark Freidlin)

\begin{abstract}
We announce a deterministic analog of Bartlett's displacement theorem. The result is that a Poisson property is stable with respect to deterministic Hamiltonian displacements. While the random point configurations move according to an $n$-body evolution, the mean measure $P$ satisfies a nonlinear Vlasov type equation $\dot{P}+y \cdot \nabla_{x} P-\nabla_{y} \cdot E(P)=0$. Combined with Bartlett's theorem, the result generalizes to interacting Brownian particles, where the mean measure satisfies a McKean-Vlasov type diffusion equation $\dot{P}+y \cdot \nabla_{x} P-\nabla_{y} \cdot E(P)-c \Delta P=0$.
\end{abstract}

\section{Poisson PROCESSES}

Some families of distributions in probability theory are natural because they appear in central limit theorems, because they maximize entropy and because they are invariant under convolutions. An example is the Gaussian distribution on $\mathbb{R}$ : adding and then normalizing independent random variables of the same distribution give a new distribution with larger relative entropy except at the attractive Gaussian fixed point of this renormalization map. A similar fixed point on discrete distributions is the Poisson distribution on $\mathbb{N}$. While the Gaussian distribution gives rise to stochastic processes like Brownian motion, the Poisson distribution occurs in random point processes like the Poisson process which is defined as follows: let $S$ be some Euclidean space and let $P$ be a finite measure on $S$. A Poisson process consists of a collection of random finite sets $\Pi(\omega)$ for which the random variables $N_{B}(\omega)=|\Pi(\omega) \cap B|$ counting the relative number of points in a Borel set $B \subset S$ are Poisson distributed with mean $P[B]$ and such that $N_{B_{j}}$ are independent for disjoint sets $B_{j} \subset S$. The mean measure $P$ determines the process because a typical point set $\Pi(\omega)$ is obtained by picking randomly a natural number $d=d(\omega)$ with probability $e^{-P[S]}(d !)^{-1}$, choosing then independently $d$ random points $a_{1}(\omega), \ldots, a_{d}(\omega)$ from $S$ with law $P$ and forming the set $\Pi(\omega)=\left\{a_{1}(\omega), \ldots, a_{d}(\omega)\right\}$. We call $P(\omega)$ the counting measure on the finite set $\Pi(\omega)$. It satisfies $P(\omega)[B]=N_{B}(\omega)$.

Poisson processes occur frequently in applications, for example as models for traffic on freeways, stars in galaxies or populations of plants. What happens if the point sets are evolved in time possibly with interaction? To have the displaced process also as a Poisson process is useful: the mean measure $P^{t}$ at a later time

Received by the editors July 28, 1997.

1991 Mathematics Subject Classification. Primary 58F05, 82C22, 60G55; Secondary 70H05, 60K35, 60J60.

Key words and phrases. Hamiltonian dynamics, Vlasov dynamics, Poisson point process. 
determines how a typical point configuration $\Pi^{t}(\omega)$ looks like. With a law for the evolution of $P^{t}$, one can disregard the possibly complicated microscopic motion of $\Pi^{t}(\omega)$ and still have the information which is needed at any time. For example, a typical star distribution of a galaxy at a later time could be determined from $P^{t}$ without integrating the Newton equations of the individual stars.

\section{The Hamiltonian displacement theorem}

A known stability of the Poisson process is when each particle is dislocated independently of the other particles. This is the displacement theorem of Bartlett, formulated in more generality in [2]. An example is when the distribution of the displaced position of a point is determined by a Markov transition probability density function $\rho^{t}(x, y)=\rho^{t}(x-y)$. The mean measure of the displaced Poisson process satisfies then $P^{t}=P \star \rho^{t}$.

The independence of the dislocations cannot be weakened in all generality as is indicated in [2]. The next theorem assures that the Poisson property is robust under deterministic Hamiltonian evolutions with nice smooth potentials $V$ for which one has global existence of the dynamics.

Theorem 2.1 (The Hamiltonian displacement theorem). Given a smooth finite measure $P$ of compact support in the phase space $S=T^{*} M$ of $M=\mathbb{R}^{q}$. It defines a random Poisson process. Assume the random finite point sets $\Pi(\omega)=$ $\left\{\left(x_{1}, y_{1}\right), \ldots,\left(x_{d}, y_{d}\right)\right\} \subset S$ are evolved with a smooth deterministic Hamiltonian d-body dynamics

$$
\dot{x}_{j}=\dot{y}_{j}, \dot{y}_{j}=-d^{-1} \sum_{k \neq j} \nabla V\left(x_{j}-x_{k}\right) .
$$

Then the displaced process does not produce correlation forces.

The probability space of the Poisson process is the big phase space $\Omega=\bigcup_{d=0}^{\infty} S^{d}$ with Borel $\sigma$-algebra and probability measure $Q=\sum_{d=0}^{\infty} e^{-P[S]}(d !)^{-1} P_{d}$, where $P_{d}=P \times \cdots \times P$ is the product measure of the multi-particle space $S^{d}$. For $\omega \in S^{d} \subset \Omega$ one gets $\Pi(\omega)=\left\{\omega_{1}, \ldots, \omega_{d}\right\}$.

The Hamiltonian displacement theorem extends to more general cases. Particles can have different masses, interact with different type of potentials, move on more general manifolds with possible boundaries, have time-dependent potentials, evolve with more general Hamiltonians, and move with a nearest neighbor interaction; they can additionally be exposed to external fields or interact with suitable $k$-body interactions. One can also add dissipation or evolve with a relativistic particle interaction.

\section{The evolution of the mean measure}

The Vlasov equation or collisionless Boltzmann equation

$$
\dot{P}^{t}+y \cdot \nabla_{x} P^{t}-E\left(x, P^{t}\right) \cdot \nabla_{y} P^{t}=0
$$

with $E\left(x, P^{t}\right)=\int_{S} \nabla V\left(x-x^{\prime}\right) d P^{t}\left(x^{\prime}, y^{\prime}\right)$ is a fundamental evolution equation in stellar dynamics or plasma physics (see [3], [1]). It has also applications in nuclear physics or in models of supersonic flows. It is a nonlinear partial differential equation. While it is usually used to evolve continuous measures, it can describe the evolution $P^{t}$ of any measure on the phase space $S$. For example, if $\tilde{P}^{t}(\omega)$ is the 
counting measure on a finite point set $\Pi^{t}(\omega)$ which evolves with the Hamiltonian $d$ body evolution with potential $V$, then $\tilde{P}^{t}(\omega)$ solves weakly the Vlasov equation. We use the tilde in order to distinguish the measure $\tilde{P}^{t}(\omega)$ coming from the Hamiltonian evolution and the random measures $P^{t}(\omega)$ defined by the Poisson process.

Theorem 3.1. The mean measure $P^{t}$ of the displaced process satisfies a nonlinear Vlasov type equation

$$
\dot{P}^{t}+y \cdot \nabla_{x} P^{t}-\nabla_{y} \cdot E\left(P^{t}\right)=0
$$

where $E\left(P^{t}\right)=\int_{\Omega} E\left(x, P^{t}(\omega)\right) P^{t}(\omega) d Q(\omega)$ with

$$
E\left(x, P^{t}(\omega)\right)=P^{t}(\omega)[S]^{-1} \int_{S} \nabla V\left(x-x^{\prime}\right) d P^{t}(\omega)
$$

and where $P^{t}(\omega)$ are the random measures defined by $P^{t}$. The measure $E\left(P^{t}\right)$ has a smooth density and is defined by $P^{t}$ alone.

The nonlinear evolution equation (3.1) encodes the statistics of the finite dimensional particle evolutions $\Pi^{t}(\omega)$ similar as diffusion equations encode stochastic particle motions. The mean Vlasov equation is an average of Vlasov equations for the discrete measures $\tilde{P}^{t}(\omega)$. It approaches the Vlasov equation for the smooth measure in the limit when the particle density converges to infinity. The mean measure $P^{t}$ would also satisfy the actual Vlasov equation if each particle configuration $\Pi(t)$ would move under the averaged field $E(x, P)$ of the mean measure $P$. In this case, the displacement result is an easy consequence of the mapping theorem in the theory of Poisson processes [2]: if $X^{t}$ is the symplectic transformations on $S$ satisfying the characteristic equations so that $X_{*}^{t} P^{0}=P^{t}$ solves the Vlasov equation, then the process at time $t$ would be the image of the process at time $t=0$ under the map $X^{t}$.

\section{INTERACTING BRoWNiAn PARTICLES}

The Hamiltonian displacement theorem is related to the already mentioned displacement theorem of Bartlett which is the same statement under the assumption that each particle is displaced independently of the other particles without interaction. For example, if each particle moves along an independent Brownian path then the displaced process stays a Poisson process and the mean measure $P^{t}$ satisfies the heat equation $\dot{P}=c \Delta P$.

The Hamiltonian displacement theorem can be combined with Bartlett's theorem and applied to Hamiltonian $d$-body dynamics driven by white noise. The Hamilton equations are now replaced by the stochastic differential equations

$$
d x_{j}=y_{j} d t, \quad d y_{j}=-\frac{1}{d} \sum_{k \neq j} \nabla V\left(x_{j}-x_{k}\right) d t+c d B_{j},
$$

where $\left\{B_{j}\right\}_{j=1}^{d}$ is a collection of independent Brownian motions and $c$ is a real parameter.

Theorem 4.1. Assume the point configurations $\Pi(\omega)$ of a Poisson process evolve as interacting Brownian particles; then the displaced process has a correlation measure which satisfies a McKean-Vlasov diffusion equation

$$
\dot{P}+y \cdot \nabla_{x} P-\nabla_{y} \cdot E(P)-\frac{c^{2}}{2} \Delta P=0
$$


which becomes the mean Vlasov equation in the limit $c=0$ when the particles evolve as a Hamiltonian system.

Adding to the Hamiltonian evolution the dynamics of interacting Brownian particles provides according to Nelson's stochastic mechanics a quantization of classical mechanics. Mathematically, the limit $c \rightarrow 0$ could serve as a technical regularization tool to investigate the mean Vlasov equation. As in the case $c=0$, one obtains in the limit of an infinite particle density the McKean-Vlasov equation

$$
\dot{P}+y \cdot \nabla_{x} P-E(x, P) \cdot \nabla_{y} P-\frac{c^{2}}{2} \Delta P=0
$$

which is for $c=0$ the Vlasov equation.

\section{TO the PRoof of the Hamiltonian displacement theorem}

In this section we say something about the proof of the Hamiltonian displacement theorem.

Rényi's theorem in the theory of Poisson processes [2] states that if $Q\left[N_{B}=0\right]$ $=\exp (-P[B])$ for all finite unions of cubes $B$, then $N_{B}$ is defined by a Poisson process. This result, obtained by Rényi in 1967, simplifies the task to verify the conditions for the Poisson process. Especially, it frees us from the duty to check the independence property.

Let $X^{t}(\omega): S \rightarrow S$ be the one-parameter family of symplectomorphisms on $S$ defined by $X^{t}(\omega)=\left(f^{t}(\omega), g^{t}(\omega)\right)$ which satisfy the Hamilton equations

$$
\dot{f}^{t}(\omega, x, y)=g^{t}(\omega, x, y), \quad \dot{g}^{t}(\omega, x, y)=-E\left(f^{t}(\omega), P^{0}(\omega)\right)(x, y) .
$$

These equations are the characteristic equations of the Vlasov dynamics and the push-forwards $\tilde{P}^{t}(\omega)=X_{*}^{t}(\omega) P^{0}(\omega)$ solve weakly the Vlasov equation.

Consider a family of Poisson processes with mean measure $P^{t}$, where $P^{t}$ satisfies the mean Vlasov equation. To prove the theorem, we compare the random point process

$$
X^{t}(\omega) \Pi^{0}(\omega)
$$

with the Poisson point process $\Pi^{t}(\omega)$. The main task is to show

$$
\frac{d}{d t} Q\left[N_{X^{-t}(\omega)(B)}(\omega)=0\right]=\frac{d}{d t} \exp \left(-P^{t}[B]\right)
$$

for any finite union of cubes $B \subset S$. With this and with Rényi's result the displacement theorem follows.

\section{REFERENCES}

1. L. A. Bunimovich et al., Dynamical systems II, Encyclopaedia of Mathematical Sciences, vol. 2 (Ya. G. Sinai, ed.), Springer-Verlag, Berlin, 1989. MR 91i:58079

2. J. F. C. Kingman, Poisson processes, Oxford Studies in Probability, vol. 3, Clarendon Press, Oxford University Press, New York, 1993. MR 94a:60052

3. H. Spohn Large scale dynamics of interacting particles, Texts and monographs in physics, Springer-Verlag, New York, 1991.

Department of Mathematics, University of Arizona, Tucson, AZ 85721

Current address: Department of Mathematics, University of Texas, Austin, TX 78712

E-mail address: knill@math.utexas.edu 